\title{
Scheduling Algorithm for Real-time VBR Video Streams Using Weighted Switch Deficit Round Robin
}

\author{
Min Chen and Gang Wei \\ Dept. of Electronic and Communication Engineering, South China University of Technology \\ chenminnew@21cn.com andecgwei@scut.edu.cn
}

\begin{abstract}
The paper presents a modified version of the deficit round-robin (DRR) scheduling algorithm due to [1]. The proposed scheduling scheme called Weighted Switch Deficit Round Robin (WSDRR) categorises the different frame types of compressed video which are handled in separate queues and prioritizes time-sensitive and visually important data. WSDRR also introduces an overdraft threshold that basically allows a packet to be scheduled even if its flow's deficit is not large enough in a given scheduling round. By numerical experiments, WSDRR achieves better delay and delay jitter performance than DRR scheduling algorithm. Hence the delay, jitter can further meet requirement for real-time $V B R$ video transmission.
\end{abstract}

\section{Introduction}

Video applications have stringent delay and delay jitter requirements which may not be met in best-effort networks. Scheduling mechanisms to be deployed in the Internet are crucial to satisfy such requirements in DiffServ (Differentiated Services) networks.

The topic of video transmission over DiffServ networks has been discussed in some papers [2], where proposed some video marking algorithm that marking the packets at the video server before transmission according to their contribution to the perceived picture quality. If the packet is marked as no important, then it will be dropped first in case of congestion. We set up a DiffServ network model, video sub-streams are classified into AF1 or AF2 class according to their contribution to the perceived picture quality, while all the background traffic is classified into $\mathrm{BE}$ class. In the border router, a weighted switch deficit round robin scheduler for the AF (Assured Forwarding) classes [3] is implemented.

The idea of priority/grouping to improve the decisions about which packets to discard has been around for a long time. The DiffServ models have been developed by numerous researchers. In addition, both deficit allocation and dynamic round robin schedulers have been developed by others [4 6], but we specially introduce overdraft service quanta to DRR scheduler. The other contribution here is the combination of above two ideas and the simulation study that illustrates the effectiveness of the combined ideas.

\section{Weighted Switch Deficit Round Robin}

The idea in DRR [1] is that, during a given busy period for a given flow, the unused portion (deficit) of the per-round bandwidth allocation (bit allowance) rolls over to the next round. Consequently, a flow that is shortchanged in a particular round can be compensated in the next round. However, there has to be enough deficit accumulation prior to servicing a large packet by this mechanism, and latency problems arise if and when the busy periods for a given flow start with large packets, especially for some video streams having known variation (e.g. I-frame) that are large but last for short periods of time. Beyond protecting these delay sensitive flows through allocating high weighted bandwidth to their queue, we also dynamically adjust the quantum of service. We address this issue by allowing "overdraft," i.e., borrowing against expected future deficits. With this modification, a flow can, in a particular round, exceed the available byte allowance up to a certain threshold (a fraction of the maximum packet size), thus yielding a negative deficit which is to be restored in the subsequent rounds before another large packet can be serviced.

\section{Simulation scenarios and results}

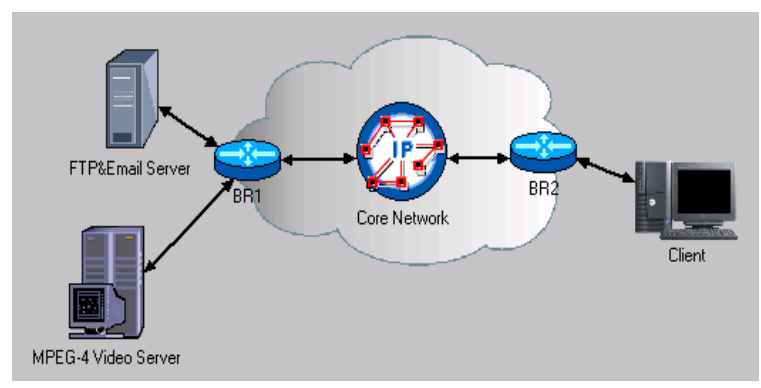

Figure 1. The topology of DiffServ network model

Our IP DiffServ network model is shown in Figure 1. It consists of a MPEG-4 video server, a FTP\&Email server and a client which are connected respectively to core network by border routers (BR1, BR2). The FTP\&Email server generates best-effort (BE) background 
traffic. The real-time video streams are produced by the MPEG-4 video server, and are encapsulated into RTP/UDP/IP packets. Among all the MPEG video streams, I frames and MV data of P frames are marked as AF1 traffic while B frames and texture data of $\mathrm{P}$ frames are classified into AF2 traffic. When packets are transmitted to the DiffServ border router, they will be classified, conditioned and scheduled. Two queues are implemented in border router to schedule different traffic, one is FIFO-queue for AF1 flows, the other is RIO-queue for AF2 and BE flows. IN/OUT checking with different token bucket mechanisms is used before a packet is queued. An AF1 packet that fails the test is lost, while an AF2 is degraded to a best effort flow. The setting of router parameters should make WSDRR effectively protect I frames and MV data of $\mathrm{P}$ frames even under heavy loads, and passing the reliability responsibility to real-time video application itself to antagonize the lost $\mathrm{B}$ frames and texture data of $P$ frames.

Using the Diffserv network model mentioned above, we have done numerical experiments on video encoding, transmission and decoding. The testing video sequence is Forman that is coded in QCIF format $(176 \times 144$ pixels/frame) at a temporal resolution of $30 \mathrm{fps}$.

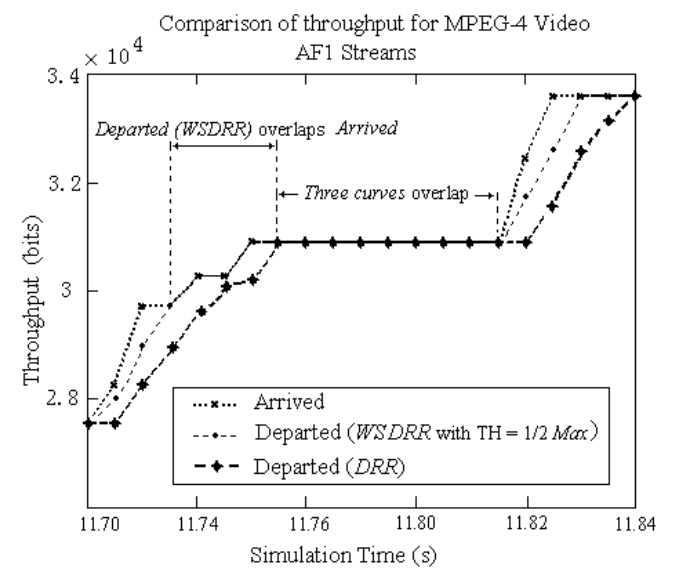

Figure 2. Comparison of throughput

To verify WSDRR achieves better delay performance than DRR, we do a group experiments with overdraft threshold (TH) equal to 0 (WSDRR degrades to weighted DRR), Max/2, Max (Maximum packet size) respectively. Fig. 2 shows a typical example of burst packet arrivals. The burst is caused by some streams having known variations that are large but last for short periods of time. In DRR, nearly constant amount of packets departs from the queue. However, in WSDRR, the number of bits departed is increased or decreased corresponding to the dynamical service quanta. In Fig2, the horizontal distance between two curves represents queuing delay in the scheduler, which corresponds to the accumulated packet delay and jitter showed in Fig.3. In addition, the vertical distance between two arrived and departed curves in Fig.2 denotes the amount of bits backlogged in the queue. Clearly, the scheduler can achieve both guaranteeing realtime service and reducing the buffer occupancy.
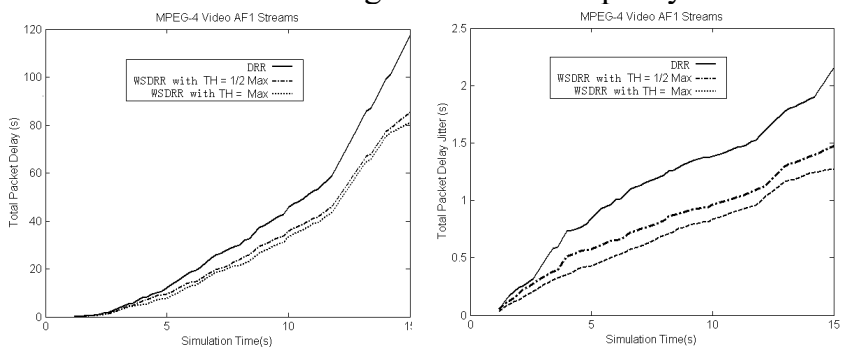

Figure 3. Comparison of Accumulated packet delay and jitter

\section{Summary}

We discussed a certain round-robin scheduling algorithm for effective and fair servicing of real-time variable bit-rate flows in IP networks. The algorithm, called weighted switch deficit round robin scheduling, dynamically adjusts service quanta based on a weighted round robin scheduling algorithm. The dynamic adjustment is based on flow-specific conditions, for example, allowing more data to be sent in anticipation that less data will be sent in the future (i.e., deficit bandwidth allocation). The idea is that some streams have known variations (e.g., I-frames) that are large but last for short periods of time. The proposed algorithms also partition data packets into high and low priority groups so drop decisions will throw away less useful packets (e.g., $\mathrm{B}$-frames and texture data of P frames in MPEG video) when resource constraints are met. Compared with DRR, WSDRR has better delay and delay jitter performance.

\section{References}

[1] M. Shreedha, G. Varghese, "Efficient fair queuing using deficit round-robin," IEEE/ACM Transactions on Networking, vol.4, Issue 3, pp. 375 -385, June 1996

[2] T. Ahmed, A. Mehaoua, G. Buridant, "Implementing MPEG-4 Video On Demand Over IP Differentiated Services," GLOBECOM 2001, vol. 4, pp. 2489-2493.

[3] J. Heinanen, F. Baker, W. Weiss and J. Wroclawski, "Assured Forwarding PHB Group," RFC 2597, IETF, June 1999.

[4] Jung-Shian Li, "An Evaluation of Deficit Round Robin Fair Queuing Applied in Router Congestion Control," Journal of Information Science and Engineering, vol. 18/2, pp. 333-339, March 2002.

[5] G. Jennes, G. Leduc and M. Tufail, "A Scheduler for Relative Delay Service Differentiation," Annals of Telecommunications, vol. 571-2, 2002

[6] M.Tufail, G.Jennes and G.Leduc, "Attaining per flow QoS with Class-based Differentiated Services," SPIE'99 